\title{
Spububsaúde
}

\section{Atuação dos auditores internos em organizações de saúde no Poder Executivo Federal Brasileiro}

\author{
The role of internal audit in Brazilian Federal Executive health entities
}

\author{
Renor Antonio Antunes Ribeiro" 0 \\ Controladoria Geral da União - CGU, Brasília, Distrito Federal, Brasil. *Autor para correspondência. E-mail: renor.ribeiro@gmail.com
}

\begin{abstract}
Resumo: O artigo analisa o alinhamento da atuação do auditor interno com as normas internacionais, nomeadamente às declarações de posicionamento da auditoria interna, do Instituto dos Auditores Internos - IIA. Os materiais e métodos foram os padrões internacionais para a gestão de riscos, para os modelos de governança e para a atuação auditoria interna, a legislação federal brasileira atinente à gestão de riscos e a leitura e análise de pesquisas publicadas sobre a atuação da auditoria interna. Os procedimentos metodológicos foram feitos por meio de pesquisa bibliográfica e coleta de dados empírica, através de entrevistas semiestruturadas e gravadas em aúdio. Como resultado da coleta de dados bibliográfica e empírica, foi possível entender as práticas de auditoria interna nas entidades federais brasileiras na área da saúde. Por fim, consideramos, com base nas normas internacionais, na revisão bibliográfica e nos estudos empíricos, que as funções desempenhadas pela auditoria interna, conforme a percepção dos participantes, está alinhada às normas e padrões internacionais aplicáveis.
\end{abstract}

Palavras-chave: saúde, pública, auditor, interno, gestão, riscos.

\begin{abstract}
The article analyzes the alignment of the performance of the internal auditor with international standards, namely the statements of positioning of the internal audit, of the Institute of Internal Auditors - IIA. The materials and methods were the international standards for risk management, for governance models and for internal auditing, the Brazilian federal legislation regarding risk management and the reading and analysis of published research on the performance of internal auditing. The methodological procedures were carried out through bibliographic research and empirical data collection, through semistructured interviews and recorded in audio. As a result of the collection of bibliographic and empirical data, it was possible to understand the internal audit practices in Brazilian federal entities in the area of health. Finally, we consider, based on international norms, bibliographic review and empirical studies, that the functions performed by the internal audit, according to the participants' perception, are aligned with the applicable international norms and standards.
\end{abstract}

Keywords: public, health, auditor, internal, management, risk.

\section{Introdução}

A internacionalização das empresas tornou necessária a melhoria da governança corporativa, sendo a gestão de riscos um dos seus componentes. Além disso, para as entidades do setor de saúde, uma boa gestão pode contribuir para salvar vidas e otimizar os custos dos serviços. Com isso, a gestão de riscos pode ser considerada uma ferramenta fundamental para a melhoria contínua dos processos de trabalho em qualquer organização de saúde.

As práticas utilizadas para a governança corporativa no setor privado estão sendo adotadas no setor público, notadamente no Poder Executivo Federal, que passou incorporar a gestão de riscos à sua base normativa, incluindo ainda o modelo das três linhas, do IIA (2020), surgindo então a necessidade de saber se a atuação da auditoria interna nas entidades de saúde do Poder Executivo Federal brasileiro está alinhada com as melhores práticas, conforme a percepção dos colaboradores dessas organizações.

Essas melhores práticas estão descritas nas normas e padrões internacionais que foram consideradas neste artigo, quais sejam: o Comitê de Organizações Patrocinadoras da Comissão Treadway - COSO; a Organização Internacional de Padronização - ISO; a Federação das Associações Europeias de Gestão de Risco - FERMA; e a Estrutura Internacional de Práticas Profissionais - IPPF, do Instituto dos Auditores Internos - IIA.

O estudo justifica-se pelo fato de que a o aperfeiçoamento contínuo da governança corporativa no setor público pode contribuir para o atendimento do direito à saúde, estatuído na carta magna brasileira de 1988, por meio da melhoria da governança dessas instituições. Com efeito, a gestão de riscos e a auditoria interna 
podem contribuir para o alcance desses objetivos, lembrando que as camadas mais vulneráveis da população brasieira só consegue ter acesso á saúde por meio dos serviços públicos.

Com o objetivo analisar o alinhamento da atuação do auditor interno com as normas internacionais, foram analisados os modelos do COSO, ISO e FERMA para os riscos e a gestão de riscos, o modelo das três linhas do IIA, a base normativa brasileira sobre o tema, além de estudos empíricos sobre o papel da auditoria interna nas organizações. Como Procedimentos metodológicos, além da pesquisa bibliográfica,foram feitas entrevistas com auditores internos de organizações federais de saúde, com a participação de oito profissionais. Após a transcrição das entrevistas gravadas com o consentimento dos participantes, foi realizada a análise dessas entrevistas, em cotejo com os autores citados na revisão bibliográfica, a qual foi estruturada nos seguintes tópicos: a implementação da auditoria interna e da gestão dos riscos; o papel da gestão de riscos e desafios; e o papel da auditoria interna na gestão de riscos e desafios. Por fim, foi feita uma analise e discussão das entrevistas e dos autores da pesquisa bibliográfica. Para avaliarmos os conteúdos dos discursos dos participantes, será necessário o estudo das normas e autores relacionados aos objetivos dessa pesquisa, os quais abordaremos por intermédio dos materiais e métodos utilizados.

\section{Materiais e métodos}

\section{Conceito de riscos e de gestão de riscos}

O risco é inerente a qualquer empresa, tendo em vista que cada ação posta em pratica implica em riscos. A ISO 31000 (2018) conceitua o riscos como sendo o “efeito da incerteza nos objetivos” (ISO 31000, 2018, p.1). Ocorre que esse efeito é expresso como um desvio dos objetivos incialmente definidos no planejamento estratégico da organização, podendo se tratar de uma oportunidade (efeito positivo) ou um risco (efeito negativo).

A gestão de riscos deve ser encarada como um processo, posto em prática, o COSO (2007) para mitigar as possibilidades de desvios significativos em relação aos objetivos. Ao auditor interno, cabe a avaliação dessa gestão de riscos. Por meio de um processo dinâmico que se aperfeiçoa constantemente, é possível que o desempenho da organização passe por um processo de melhoria contínua, de modo que a auditoria interna é a instância capaz de fornecer informações relevantes para a alta administração da entidade. Segundo Ribeiro (2020a), a gestão de riscos tem como finalidade a garantia razoável do alcance dos objetivos, de modo a reduzir os riscos residuais para níveis compatíveis com o apetite da organização.

A ISO 31000 (2018, p. 9), define o processo de gestão de riscos como a “aplicação sistemática de políticas, procedimentos e práticas de gestão envolvendo as atividades de comunicação, consulta, estabelecimento do contexto e avaliação, tratamento, monitoramento, registro e relato dos riscos”. Segundo a FERMA, na gestão de riscos, as "organizações analisam metodicamente os riscos inerentes às respectivas actividades, com o objectivo de atingirem uma vantagem sustentada em cada actividade individual e no conjunto de todas as actividades" (FERMA, 2002, p. 3). Para o COSO (2009), a gestão dos riscos deverá ser posta em prática pela alta administração, espraiando-se para todos os setores, do topo para a base, tomando como base o planejamento estratégico e identificando todos os eventos que possam afetar o alcance dos objetivos da organização.

\section{O modelo das três linhas e o papel da auditoria interna}

O IIA (2013) construiu um modelo de governança intitulado de "modelo das três linhas de defesa”, e que foi revisto em 2020, passando a se chamar de "Modelo das três linhas do IIA 2020" (IIA, 2020). Para o IIA (2020, p.3), "Os papéis de primeira linha estão mais diretamente alinhados com a entrega de produtos e/ou serviços aos clientes da organização", enquanto que a segunda linha fornece assistência especializada para os integrantes da primeira linha, abrangendo consultoria e aconselhamento sobre como a gestão de riscos pode ser melhorada na organização (IIA, 2020). Miranda (2019), indica que são os gestores os responsáveis pela primeira linha, enquanto que cabe à segunda linha o exercício de papeis de supervisão e de verificação da conformidade das instâncias de primeira linha.

A auditoria internas constitui-se na terceira linha, responsável pela avaliação dos controles internos das duas primeiras linhas. Para o IIA (2020, p.3), “a auditoria interna presta avaliação e assessoria independentes e objetivas sobre a adequação e eficácia da governança e do gerenciamento de riscos”. Para Barbosa (2020), a auditoria interna executa uma atividade com objetividade e independência, para a melhoria dos processos organizacionais, dentre os quais está a gestão de riscos. Para Ribeiro $(2020, b)$, a auditoria interna deve avaliar a gestão de riscos da entidade, para ver se os controles internos adotados estão mitigando os riscos inerentes 
para níveis aceitáveis. Com efeito, a auditoria interna é uma atividade independente, de avaliação ao conselho gestor da entidade, em relação à eficácia da gestão de riscos, oferecendo também consultoria sobre a melhoria desses processos, sem, no entanto, ser responsável pela gestão dos riscos (IIA, 2009).

\section{A base normativa sobre a gestão dos riscos no setor público federal brasileiro}

O Decreto Lei $200^{1}$, de 25 de fevereiro de 1967, que foi recepcionado pela Constituição Federal ${ }^{2}$ brasileira de 1988, discorreu sobre risco e controle nos artigos 13 e 14, estabelecendo, em seu artigo 13, uma estrutura de governança compreendendo: "o controle pela chefia compentente; o contrôle pelos órgãos próprios de cada sistema; e o contrôle pela contabilidade e auditoria”. Em 2016 entraram em vigor, no Brasil, os seguintes diplomas legais: a Instrução Normativa Conjunta MP/CGU n ${ }^{\circ} 01$, de 10 de maio de $2016^{3}$, que disciplinou, para o Poder Executivo Federal, os controles internos, gestão dos riscos e governança; a Lei $n^{\circ} 13.303$, de 30 de junho de $2016^{4}$, com regras de gestão de riscos, aplicável para as empresas públicas, sociedade de economia mista e suas subsidiárias, em todos os poderes e entes federativos brasileiros; e o Decreto $\mathrm{n}^{\circ} 8.945$, de 27 de dezembro de $2016^{5}$, que regulamentou a Lei $13.303 / 16$ para os órgãos e entidades da União.

\section{A Atuação da auditoria interna}

A atuação da auditoria interna, tanto nas normas internacionais, quanto no seio das organizações, tem contribuido para a melhoria dos processos internos, em benefício da organização. Nesse sentido, o estudo de Macena et al. (2017), destacou que o funcionamento da auditoria interna em uma entidade privada do setor hospitalar, foi motivada incialmente pelo corte de despesas, mas que também ajudou a melhorar a gestão da organização. Mas a unidade de auditoria interna não se presta somente para a melhoria dos processos, tendo em consideração que Borges et al. (2015) observaram que os auditores internos contribuiram também para a melhoria da qualidade no atendimento aos pacientes.

Ao aplicarem um questionário a (47) empregados de uma das maiores organizações hospitalares da República Checa, Keclíková \& Briš (2011), mostraram que os auditores internos são fundamentais na avaliação da gestão de riscos, entendendo que os processos de gestão de riscos podem melhorar os níveis de segurança dos usuários dos serviços.

Os relatórios e recomendações que a auditoria interna produz, são de fundamental importância para a melhoria da gestão. Nesse sentido, Trisciuzzi (2009) destaca as recomendações estruturantes, que apontam aperfeiçoamentos a serem feitos no sistema de controle interno (SCI), oferecem subsídios à gestão no atingimento dos objetivos. Por meio do estudo em quinhentas empresas de Portugal, Morais (2008), observou que as recomendações da auditoria interna são usadas para a melhoria da gestão nas organizações pesquisadas e que são requeridos serviços de aconselhamento e consultoria.

A auditoria interna também pode contribuir para a obediência dos processos de trabalho aos normativos legais. Soares (2007), ao investigar uma instituição de saúde brasileira, observou que a auditoria interna avalia a conformidade do SCI com os dispositivos legais, o que é fundamental para uma organização filantrópica. Duarte (2017), ao investigar cinco hospitais públicos, complementa que a terceira linha é uma mais valia não somente para a melhoria do SCI, mas também para promover a eficiência na gestão dos recursos.para promover a eficiente gestão dos recursos.

Além do mais, Silva \& Costa (2019), ao analisarem a atuação da auditoria interna em uma organização federal, apontam que a governança e auxílio à tomada de decisão são duas funções exercidas pela terceira linha, além de fornecer garantia razoável sobre o desempenho organizacional e a gestão dos riscos. Todavia, Brito et al. (2017) diz que a estrutura de gestão de riscos é um dos fatores de sucesso do gerenciamento dos riscos.

Em virtude dos argumentos apresentados, iremos tratar, nos itens seguintes, dos procedimentos metodológicos para o presente estudo, dos procedimentos metodológicos, para entendermos como foi feita a coleta e a análise dos dados para as oito organizações da área de saúde, de forma a vermos qual foi, segundo a percepção dos participantes, a atuação da auditoria interna nessas organizações.

\footnotetext{
${ }^{1}$ http://www.planalto.gov.br/ccivil_03/decreto-lei/del0200.htm

${ }^{2} \mathrm{http}: / /$ www.planalto.gov.br/ccivil_03/constituicao/constituicao.htm

${ }^{3}$ https://www.in.gov.br/materia/-/asset_publisher/Kujrw0TZC2Mb/content/id/21519355/do1-2016-05-11-instrucao-normativa-conjunta-n-1-de-10-demaio-de-2016-21519197

${ }^{4}$ http://www.planalto.gov.br/ccivil_03/ato2015-2018/2016/lei/113303.htm

${ }^{5}$ http://www.planalto.gov.br/ccivil_03/_Ato2015-2018/2016/Decreto/D8945.htm
} 


\section{Procedimentos metodológicos}

Como procedimentos metodológicos, foram adotados a revisão bibliográfica e da coleta de dados de forma empírica, em que os participantes concordaram em ter suas falas gravadas, para melhor compreensão do tema estudado (Vergara, 2009). Hill \& Hill (2016) acrescentam que para uma melhor compreensão do tema, é necessária a observação, sendo que Denzin \& Lincoln (2006), ponderam que, na pesquisa qualitativa, a atenção deve se voltar para o ambiente em que o processo está ocorrendo, para seu entendimento.

As entrevistas realizadas neste estudo foram semi estruturadas, sendo que a pesquisa foi qualitativa. Conforme Gil (2008), nas pesquisas qualitativas, as entrevistas podem ser utilizadas para se aprofundar um tema. A gravação da entrevista foi utilizada para que o entrevistador se preocupasse em conduzir o processo junto com o entrevistado. Segundo Edwards \& Holland (2013) o uso do dispositivo de gravação libera o entrevistador da obrigação de fazer anotações, fazendo com que ele se concentre em interagir, estimular e ouvir o participante. Entretanto, segundo Belei et al. (2008), essa iteração não pode influenciar no discurso do entrevistado. Mesmo assim, a entrevista é uma técnica que pode novos elementos para a pesquisa, de acordo com Mattos \& Goldenberg (2004), sendo que isso depende tanto da habilidade do entrevistador, quanto da disposição do entrevistado em revelar detalhes sob o assunto em análise.

De modo a analisarmos de forma mais aprofundada as entidades em que os entrevistados se dispuseram a contribuir com os seus relatos, foi escolhido o estudo de casos. Mesquita \& Matos (2014), asseveram que, quanto o objeto de estudo é analisado mais profundamente, deve ser utilizado o estudo de caso, sendo que as técnicas que se destacam são a entrevista e a observação. Gil (2008), defende que o estudo de caso deverá ocorrer empiricamente, para compreensão do contexto real do fenômeno em estudo, com o auxílio de várias fontes de pesquisa.

A organizações escolhidas para o estudo foram aquelas em que os auditores internos concordaram em participar, no total de oito entrevistados, entre 28/03/2019 a 29/07/2019, abrangendo o coordenador geral de auditoria da saúde na Controladoria Geral da União - CGU, o coordenador adjunto da saúde na CGU na área de saúde, além de seis (6) auditores chefes de seis hospitais universitários federais, a partir de pesquisa tanto no sítio da CGU (https://www.gov.br/cgu/pt-br) quanto no sítio eletrônico da Empresa Brasileira de Serviços Hospitalares - EBSERH (https://www.gov.br/ebserh/pt-br). Os detalhes dos cargos e funções dos entrevistados, segregados por setor de atividade encontram-se elencados no quadro que se segue:

Tabela 1. Pessoas entrevistadas no Brasil.

\begin{tabular}{|c|c|c|}
\hline Setor de Atividade & Função ocupada pelos entrevistados no Brasil & Total de pessoas entrevistadas \\
\hline
\end{tabular}

Coordenador Geral da Auditoria Interna da CGU na Área da Saúde (P1)

Saúde

Saúde (P1)

Coordenador Geral adjunto da Auditoria Interna da CGU na Área da Saúde (P2)

\begin{tabular}{|c|c|c|c|}
\hline \multirow{6}{*}{ Hospitalar } & Auditor Chefe (P11) & \multirow{6}{*}{6} & \multirow{6}{*}{$75 \%$} \\
\hline & Auditor Chefe (P12) & & \\
\hline & Auditor Chefe (P13) & & \\
\hline & Auditor Chefe (P14) & & \\
\hline & Auditor Chefe (P15) & & \\
\hline & Auditor Interno (P16) & & \\
\hline TOTAL & & 8 & $100 \%$ \\
\hline
\end{tabular}

Nota: dados recolhidos em entrevistas aplicadas aos gestores e aos auditores internos do setor público do Brasil, em 2019.

De modo a mitigar o risco de erros ou interpretações errôneas, sendo possível a obtenção de informações adicionais, conforme observado por Guerreiro et al. (2015), ocorreu a transcrição das falas e envio das transcrições, via e-mail, aos participantes.

Em seguida foi feita a análise do material transcrito, para melhor categorização dessas falas. Bardin (1977), defende que essa análise abrange a leitura em detalhes de todo o material transcrito, com a separação de palavras, expressões e temas que tenham relação com a investigação, seguindo-se a categorização desses tópircos em categorias semânticas e sintáticas. 
Conforme Bardin (1977), a análise de conteúdo inicia-se com uma análise prévia e exploração do material, passando pelo seu tratamento dos resultados, visando a interpretação do conjunto de informações categorizadas. Embora as transcrições tenham sido feitas de forma literal, para efeitos de inclusão neste trabalho, ocorreu sua edição, de forma a corrigir erros nas falas. Essa edição para corrigir erros e vícios de linguagem é defendida por Duarte (2004), embora, segundo Azevedo et al. (2017), as transcrições iniciais possam ser feitas de forma literal ou preservando o seu conteúdo. Por fim, foi realizada a análise de conteúdo, definida por André (1983), com a redução de um grande volume de material em várias categorias.

\section{Resultados e discussão}

A revisão da literatura, bem como a análise da base normativa e dos dados empíricos, possibilitou o entendimento da atuação da terceira linha nas oito entidades de saúde federais. De modo a se compreender, segundo os relatos dos participantes, essa atuação da auditoria interna, as entrevistas foram conduzidam segundo os seguintes tópicos: a implantação ou fase de implantação da auditoria interna e da gestão de riscos; qual o papel da gestão de riscos e desafios; como, de fato, a auditoria interna atua nessas organizações e os desafios a serem enfrentados.

Em termos gerais, foi observado que os participantes possuem narrativas alinhadas com o disposto nas normas internacionais e na legislação federal brasileira. Obedecendo aos tópicos levantados em cada entrevista, classificaremos as respostas dos participantes de acordo com o que se segue.

\section{Como as entidades analisadas implantaram a auditoria interna e a gestão de riscos}

Foi observado que o órgão das administração direta da saúde no Brasil não possui órgão auditoria interna. Destaque-se que, no setor de saúde, bem como em outros da administração direta, a auditoria interna é a própria CGU, órgão responsável pelo SCI do Poder Executivo Federal brasileiro - PEF (TCU, 2018). A esse respeito, P2 relatou que a gestão de riscos não foi implementada, fato confirmado por P1 e P5. O TCU (2018) destacou que as auditorias internas responsáveis por algumas entidades avaliadas, a exemplo do setor da saúde, estão em um nível incipiente da avaliação de riscos e controles voltados à corrupção (TCU, 2018; Acórdão TCU n 2604/2018 - Plenário). Júnior et al. (2015) entendem que há poucos órgãos com políticas ou práticas de gestão de riscos formalizadas no Brasil.

Além disso, P1 e P2 relataram que o setor de saúde brasileiro estava numa etapa incipiente de implementação de um processo de gestão de riscos. Todavia, nos hospitais universitários, conforme o relato de P3, P4, P5,P6, P7 e P8, a gestão de riscos está em fase de implementação ou já implementada. Dessa forma, temos duas situações distintas: uma em que o órgão de saúde não possui auditoria interna e também encontra-se em um nível incipiente de implementação da gestão de riscos; a outra, em que os hospitais universitários possuem auditorias internas e gestão de riscos implementada ou em implementação. Isso implica que, de acordo com Trisciuzzi (2009), que as organizações que não possuem auditoria interna em suas entidades estão perdendo valiosos benefícios proporcionados por essa instância de controle, além de correrem riscos de confiar unicamente na gerência, que pode não ter a independência necessária para opinar sobre sua própria gestão, como seria de se esperar da auditoria interna. Em complemento, Silva e Costa (2019) defendem que uma auditoria interna dentro da estrutura de uma entidade pode contribuir para a gestão.

\section{Papel da gestão de riscos e desafios}

Os participantes apresentaram discursos alinhados as normas internacionais, nomeadamente as do IPPF, especialmente em relação as declarações de posicionamento sobre as três linhas e ao papel da auditoria interna na gestão de riscos das organizações. Assim, eles entendiam e relatavam que cumpriam esses papeis, demonstrando aos gestores, sempre que possível, os seus papeis e funções dentro do SCI das respectivas organizações.

Esses relatos também estavam alinhados às disposições da legislação federal brasileira e da ISO, da FERMA, do IIA e do COSO-ERM. Nesse sentido, Brito et al. (2017) ponderam que a estrutura de gestão de riscos está diretamente relacionada ao sucesso da auditoria interna com base nos riscos. Mustapha \& Abidin (2017) observam que o trabalho da auditoria é facilitado pela presença da gestão de riscos na organização.

\section{Como as auditorias internas atuam e quais são os desafios a serem enfrentados}

De acordo com os relatos dos participantes, a atuação da auditoria interna abarca a avaliação da eficácia do SCI e da gestão de riscos das organizações em que atuam. Os participantes entendem que a auditoria 
interna não é responsável pelo gerenciamento dos riscos e sim pela avaliação dessse gerenciamento. O papel de gerir os riscos pertence, segundo os relatos dos entrevistados, à primeira e à segunda linhas.

Sobre o papel de consultoria, realizado pela auditoria interna no hospital universitário onde atua, P3 relatou que: nós fizemos um trabalho de começar a trabalhar essa cultura de riscos com os empregados. para fazer com que eles identificassem quais eram os principais riscos. No segundo ano nós fizemos uma auditoria, que avaliou a adequação desses riscos.

Para P6, no âmbito de sua entidade, a gestão de riscos tem como principal desafio a implementação sistemática em toda a organização e não apenas em alguns setores, de forma isolada. Conforme o relato do entrevistado:

Nós devemos estar aí no meio do caminho e vejo que a maior dificuldade é transformar a gestão de riscos num processo corporativo e não em iniciativas localizadas (...). Dentre os processo licitatórios, temos a gestão de riscos por força de lei, e temos gestão de riscos nas áreas assistenciais, por força de lei e também pelo nível de criticidade do paciente. Mas eu acho que a alta administração da empresa tem que se apropriar desse processo de forma sistêmica e corporativa.

Assim sendo, os relatos dos participantes destacaram a compreensão acerca do papel da auditoria interna na avaliação da gestão organizacional, incluindo aí a gestão dos riscos. Alexandre (2016, p. 55), também observou, nos hospitais públicos de Portugal, que a auditoria interna contribui para "a Gestão de Risco, o reforço do SCI, o combate à fraude, a criação de valor e o apoio à gestão [. . . ] indo de encontro aos objetivos previstos para a função".

Os auditores internos dos hospitais federais apresentaram relatos alinhados com as normas internacionais sobre o âmbito da atuação da auditoria interna, sobre o SCI e sobre o processo de gestão de riscos e, além de demonstrarem assumir uma postura proativa, por meio tanto de cursos e treinamentos relacionados ao tema, quanto por meio da troca de ideias entre os colegas de outras organizações. Observou-se ainda uma vantagem do papel de consultoria/aconselhamento em relação ao papel de avaliação. A esse respeito, devemos considerar a implantação recente da IN MP/CGU n ${ }^{\circ}$ 01/2016, a qual entrou em vigor praticamente dois anos antes da realização das entrevistas, de modo que gestores e funcionários da primeira e segundas linhas, das respectivas organizações, necessitariam de cursos e consultorias para disseminar o processo, tecnicas e a cultura da gestão de riscos.

\section{Considerações finais}

Conforme levantamentos de dados bibliográficos e informações das entrevistas realizadas nas organizações federais de saúde, observou-se que a auditoria avalia o SCI e oferece serviços de aconselhamento nas entidades de saúde analisadas, em consonância aos normativos internacionais referentes ao modelo das três linhas de defesa do IIA (2013) e aos papeis da auditoria interna na gestão de riscos (IIA, 2009). Nos setores auditados pelo órgão central de auditoria interna do Poder Executivo Federal brasileiro, os quais correspondem aos órgãos federais da administração direta da União que não possuem auditoria interna em sua estrutura organizacional, a gestão de riscos está em grau incipiente.

Conforme destacado, uma auditoria interna dentro da estrutura da organização parece facilitar a implantação de uma cultura de riscos. Assim, a presença de auditor interno da própria estrutura organizacional,concorrentemente com a CGU, parece facilitar a informação e comunicação, o que pode ajudar a disseminar uma cultura de riscos, o que, segundo relatos dos participantes, parece ser mais difícil de implementar em ambientes com menor presença de auditores governamentais. No caso de entidades que possuem apenas a CGU como auditoria interna, os participantes relataram que a gestão de riscos encontra-se em uma fase inicial. Porem, nos hospitais federais, todos com serviços de auditoria interna dentro de sua estrutura, a gestão de riscos tende a ser mais implementada e há mais conversas com os gestores sobre os riscos e sua gestão, segundo as narrativas dos auditores entrevistados.

Em vista disso, os auditores internos que fazem parte do quadro funcional das entidades em que atuam parecem tem mais condições de exercer seu papel para o efeitvo funciomanento da gestão de riscos do que aqueles que atuam fora da organização auditada. Portanto, a promoção de uma cultura de riscos, segundo os relatos dos entrevistados, parece estar diretamente relacionada a presença da terceira linha dentro do SCI da entidade, conforme estabelecido no modelo das três linhas do IIA. 


\section{Referências}

ABNT NBR ISO 31000. 2018. Gestão de riscos - diretrizes - risk management - guidelines. Rio de Janeiro, RJ: Associação Brasileira de Normas Técnicas

Abreu Soares, M. 2007. Auditoria interna aplicada em uma instituição filantrópica hospitalar. ConTexto, 7(11).

Alexandre, V. M. 2016. A função de Auditoria Interna no Serviço Nacional de Saúde. Dissertação de Mestrado. Lisboa, PT: Universidade Autônoma de Lisboa.

André, M. E. D. A. 1983. Texto, contexto e significados: algumas questões na análise de dados qualitativos. Cadernos de Pesquisa, 45, 66-71.

Azevedo, V., Carvalho, M., Costa, F. F., Mesquita, S., Soares, J., Teixeira, F., \& Maia, A. 2017. Transcrever entrevistas: questões conceptuais, orientações. Revista de Enfermagem Referência, 4(14), 159-168.

Barbosa, E. G., Dantas, J. A., \& Santos, D. N. 2020. Gestão dos Recursos de Tempo de Auditoria: Modelo Matemático de Estimação e de Controle. Revista TCU, 145, 30.

Bardin, L. 1977. Análise de Conteúdo. Lisboa, PT: Edições 70.

Belei, R. A., Gimeniz-Paschoal, S. R., Nascimento, E. N. \& Matsumoto, P. H. V. R. 2008. O uso de entrevista, observação e videogravação em pesquisa qualitativa. Cadernos de Educação - FaE/PPGE/UFPel, 30, 187199.

Borges, G. R., Diel, F. J., \& Fernandes, F. C. 2015. A Contribuição da Auditoria Interna de Riscos para o Planejamento de Organizações na Área de Saúde. (M. C. Novaretti, Ed.) Revista de Gestão em Sistemas de Saúde, 4(2), 9-27.

Brito, G. C., Pimenta, D. P., Souza, E. M., \& Cruz, A. F. 2016. Benefícios e desafios na implantação da auditoria baseada em risco em instituições federais de ensino. Revista GUAL, 10(4), 109-133.

Committee of Sponsoring Organizations of the Treadway Commission [COSO]. 2007. Gerenciamento de riscos corporativos - estrutura integrada: sumário executivo. Recuperado de http:www.coso.org/documents/COSO_ERM_ExecutiveSummnary_Portuguese.pdf

Committee of Sponsoring Organizations of the Treadway Commission [COSO]. 2016. Enterprise risk management. Aligning risk with strategy and performance. (June 2016 edition). Recuperado de: https://www.coso.org/Documents/COSO-ERM-draft-PostExposure-Version.pdf. Acesso em: 3 jan. 2018.

Denzin, N. K. \& Lincoln, Y. S. 2006. Introdução: a disciplina e a prática da pesquisa qualitativa. Porto Alegre, RS: Artmed.

Drogalas, G. Eleftheriadis. O, Pazarskis, M \& Anagnostopoulou, E. 2017. Perceptions about effective risk management. The crucial role of internal audit and management. Evidence from Greece. Investment Management and Financial Innovations, 14(4), 1-11.

Duarte, M. D. S. 2017. A importância da auditoria interna para uma gestão eficiente e eficaz em instituições hospitalares do setor público.

Duarte, R. 2004. Entrevistas em pesquisas qualitativas. Educar, 24, 213-225.

Edwards, R. \& Holland, J. 2013. What is qualitative interviewing? London, UK: Bloomsbury.

Federation of European Risk Management Associations [FERMA]. Recuperado de: https://www.ferma.eu/app/uploads/2011/11/a-riskmanagement-standard-portuguese-version.pdf. Acesso em: 17 dez. 2019.

Gil, A. C. 2008. Métodos e técnicas de pesquisa social (6th ed). São Paulo, SP: Atlas.

Guerini, I. C., \& Guerini, E. 2019. Racionalização das ações de controle interno e auditoria como processo de controle na gestão da saúde em unidades hospitalares no contexto Brasileiro. Revista Brasileira de Tecnologias Sociais, 6(1), 56-69.

Guerreiro, M. S., Rodrigues, L. L. \& Craig, R. 2015. Institutional Change of Accounting Systems: The Adoption of a Regime of Adapted International Financial Reporting Standards. European Accounting Review, 24(2), 379-409.

Hill, M. \& Hill, A. 2016 Investigação por questionário Lisboa, PT: Edições Sílabo.

Instituto de Auditores Internos [IAI]. 2009. IIA position paper: the role of internal auditing in enterprise-wide risk management. Recupera de: https://na.theiia.org/standardsguidance/Public\%20Documents/PP\%20The\%20Role\%20of\%20Internal\%2 0Auditi ng\%20in\%20Enterprise\%20Risk\%20Management.pdf. Acesso em: 13 dez. 2017. 
Instituto de Auditores Internos [IAI]. 2013. As três linhas de defesa no gerenciamento eficaz de riscos e controles. Recuperado de: http://www.iabrasil.org.br/new/2013/downs/As_tres_linhas_de_defesa_Declaracao de_Posicionamento2.pdf

Instituto de Auditores Internos [IAI]. 2020. Modelo das três linhas do IIA 2020: uma atualização das três linhas de defesa.

Recuperado

de:

http://www.iabrasil.org.br/new/2013/downs/As_tres_linhas_de_defesa_Declaracao _de_Posicionamento2.pdf. Acesso em: $28 \mathrm{dez} .2020$.

Júnior, A. J. S. de O., Gomes, A. R. \& Machado, G. de V. 2015. Metodologia de auditoria com foco em processo e risco. Revista do TCU, 132, 28-37.

Lima, P. N. D. 2014. O impacto da auditoria interna no desempenho organizacional. Dissertação de Mestrado. Porto, PT: Instituto politécnico do Porto.

Macena, J. L., Jordão, C. P., Xavier, J. L. J., \& Administração, U. F. P. E. 2017. Auditoria interna: uma análise da implantação em uma instituição privada de serviço de saúde (Hospital) Internal audit: an analysis of implantation in a private institution of health services (hospital).

Mattos, P. L. C. L. D. \& Goldenberg, M. 2004. A entrevista não-estruturada como forma de conversação: razões e sugestões para sua análise. Revista de Administração Pública, 39(4), 57.

Mesquita, R. F. D. \& Matos, F. R. N. 2014. Pesquisa qualitativa e estudos organizacionais: história, abordagens e perspectivas futuras. IV Colóquio Internacional de Epistemologia e Sociologia da Ciência da Administração.

Miranda, R. F. D. A. 2019. Módulo 1-Introdução à gestão de riscos: estruturas de gerenciamento e bases normativas.

Morais, M. G. da C. T. 2008. A importância da auditoria interna para a gestão: caso das Empresas portuguesas. Portugal, $18^{\circ}$ Congresso Brasileiro de Contabilidade. Recuperado de: https://docplayer.com.br/684140-Aimportancia-da-auditoriainterna-para-a-gestao-caso-das-empresas-portuguesas.html

Mustapha, W. M., \& Abidin,. N. H. Z. 2017. Práticas de auditoria interna e gerenciamento de riscos entre universidades públicas na Malásia. Journal of Research and Practice in Public Sector Accounting and Management, 7(1), 1-14.

Ribeiro, Renor. 2020. Gestão de Riscos em Organizações Públicas: normas e padrões internacionais utilizados para a gestão de riscos, etapas do processo e análise da base normativa de Portugal e do Brasil. Lisboa, PT: Edições Exlibris.

Ribeiro, Renor. 2020. Gestão de Riscos no Setor Público: normas e padrões internacionais, análise das legislações nacionais de Portugal e do Brasil e aplicação na base normativa do setor público. Brasília, DF: Athenas Editora.

Silva, A. A \& Costa, A. J. B. 2019. A contribuição da auditoria interna para a governança: estudo de caso em uma organização social vinculada ao governo federal. Cuadernos de Contabilidad, 20(49).

Souza, A. A., de Faria Marques, A. M., de Oliveira, L. F., Glória, L. G. L., \& Braga, A. S. 2013. Análise do sistema de controle interno e da auditoria interna em hospitais públicos e filantrópicos. Tourism \& Management Studies, 3, 896-906.

Trisciuzzi, C. R. F. 2009. A Auditoria Interna como ferramenta de melhoria dos controles internos de uma organização: Estudo de caso em uma empresa do segmento industrial do Rio de Janeiro. Dissertação de Mestrado. Rio de Janeiro, RJ: Universidade do Estado do Rio de Janeiro.

Vergara, S. C. 2009. Projetos e relatórios de pesquisa em administração. São Paulo, SP: Atlas.

\section{Minicurrículo}

Renor Antonio Antunes Ribeiro. Mestre em Administração Publica com ênfase em gestão de riscos e auditoria interna pela Universidade do Minho/Portugal, certificado pela ISO 31000 pelo Risk Managent Professional, Auditor Federal de Finanças e Controle da Controladoria Geral da União - CGU, Especialista em Planejamento Educacional e em Administração Pública, Engenheiro Mecânico pela Universidade Federal do Ceará - UFC, Técnico em Gestão da Produção pelo SENAI e Técnico em Contabilidade pela SEDUC/CE. Pesquisador/investigador na área de gestão de riscos, compliance, gestão da qualidade e gestão pública. Coordena e ministra cursos e treinamentos para servidores públicos federais de diversos órgãos/instituições. Foi Coordenador pedagógico, professor, instrutor, tutor e elaborador de conteúdos para treinamentos 
presenciais e à distância na Escola de Administração Fazendária - ESAF, Auditor Interno do Estado do Ceará, Professor do Serviço Social da Indústria - SESI e Professor da Secretaria de Educação do Estado do Ceará.

Como citar: Ribeiro, R.A.A. 2021. Atuação dos auditores internos em organizações de saúde do Poder Executivo Federal Brasileiro. Pubsaúde, 5, a101. DOI: https://dx.doi.org/10.31533/pubsaude5.a101

Recebido: 13 nov. 2020.

Revisado e aceito: 23 nov. 2020.

Conflito de interesse: os autores declaram, em relação aos produtos e companhias descritos nesse artigo, não ter interesses associativos, comerciais, de propriedade ou financeiros que representem conflito de interesse.

Licenciamento: Este artigo é publicado na modalidade Acesso Aberto sob a licença Creative Commons Atribuição 4.0 (CC-BY 4.0). 